\title{
Modélisation et potentialités du chauffage solaire des sols par paillage artificiel à la Guade- loupe
}

\author{
Hervé SINOQUET, Eric MIGNARD $\left({ }^{*}\right) \&$ Raymond BONHOMME \\ I.N.R.A., Station Agropédoclimatique, Centre de Recherches des Antilles et de la Guyane, B.P. 1232, F97184 \\ Pointe-à-Pitre Cedex, Guadeloupe \\ (*) I.N.R.A., Laboratoire de Science du Sol, ENSA, Centre de Recherches de Montpellier, 9, place Viala, \\ F 34060 Montpellier Cedex
}

\begin{abstract}
La « solarisation » consiste à provoquer un échauffement du sol en appliquant un paillage artificiel à sa surface, ce qui peut permettre l'élimination d'agents pathogènes du sol.

Des mesures de température effectuées à la Guadeloupe dans des sols paillés (film plastique opaque noir, film plastique transparent et verre) ont montré que des températures maximales supérieures à $50{ }^{\circ} \mathrm{C}$ pouvaient être atteintes dans la couche superficielle en fonction de la nature du paillage et de l'état hydrique du sol. Elles sont par ailleurs fortement liées à l'intensité du rayonnement solaire.

D'autre part, un modèle numérique de comportement thermique des sols paillés a été développé et validé. Son utilisation a permis de déterminer les facteurs microclimatiques responsables de l'échauffement du sol paillé, de mettre en évidence l'importance de la résistance de contact entre le paillage et la surface du sol, et de définir l'influence de l'état hydrique pour le cas d'un sol ferrallitique de Guadeloupe.
\end{abstract}

Mots clés additionnels : température du sol, rayonnement solaire, film plastique, verre, Antilles, désinfection du sol.

Solarization consists of soil heating by application of an artificial mulch. High temperatures can be reached which reduce the incidence of soil-borne pathogens. Measurements of soil temperature, made in Guadeloupe, under different mulches (black plastic, clear plastic and glass) showed that daily maximum temperatures could be higher than $50^{\circ} \mathrm{C}$ near the soil surface. Soil temperature depended on nature of mulch and soil moisture content but the most important factor was daily solar radiation. A numerical model simulating the thermal behaviour of mulched soils was developed and verified. This model, which calculates temperature profiles in the mulched soils, showed the important role of contact resistance between mulch and soil surface, and of soil moisture content, on heating for ferrallitic soils in Guadeloupe.

Additional key words : soil temperature, solar radiation, plastic film, glass, Antilles, soil disinfection.

\section{INTRODUCTION}

L'application d'un film transparent à la surface d'un sol modifie le bilan énergétique de l'interface solatmosphère et provoque une augmentation de la température du sol. Dans certaines conditions, cet échauffement du sol est suffisant pour réduire ou éliminer divers agents pathogènes telluriques. Cette méthode de désinfection, appelée souvent "solarisation ", a été proposée par l'équipe de KATAN en Israël (KATAN et al., 1976) et s'avère efficace lorsque le rayonnement solaire incident est important.
En Guadeloupe, l'utilisation de cette technique semble tout à fait indiquée puisque, d'une part, les conditions climatiques sont favorables, et d'autre part, les ravageurs telluriques sont responsables d'importantes réductions de rendement : c'est notamment le cas en cultures maraîchères répétées sur les mêmes parcelles.

Les travaux rapportés dans cet article ont consisté à évaluer les potentialités physiques de chauffage des sols par paillage artificiel en Guadeloupe. Dans ce but, un modèle de transferts thermiques dans un sol paillé, inspiré de ceux de l'équipe de MAHRER, a été développé afin de mettre en évidence les phénomènes physi- 
ques responsables de l'échauffement du sol sous paillage. Les mesures de température effectuées dans le sol ont permis de valider le modèle et de juger de l'efficacité de la solarisation aux Antilles.

\section{MATÉRIEL ET MÉTHODES}

\section{A. Présentation du modèle (fig. 1)}

Le champ de température d'un sol paillé a été modélisé par MAHRER et son équipe. Les premiers modèles étaient unidimensionnels et ne s'intéressaient qu'aux transferts thermiques (MAHRER, 1979; MAHRER, 1980) alors que les suivants se sont compliqués par le passage à deux dimensions (MAHRER \& KATAN, 1981) puis la prise en compte des transferts d'humidité (MAHRER et al., 1984).

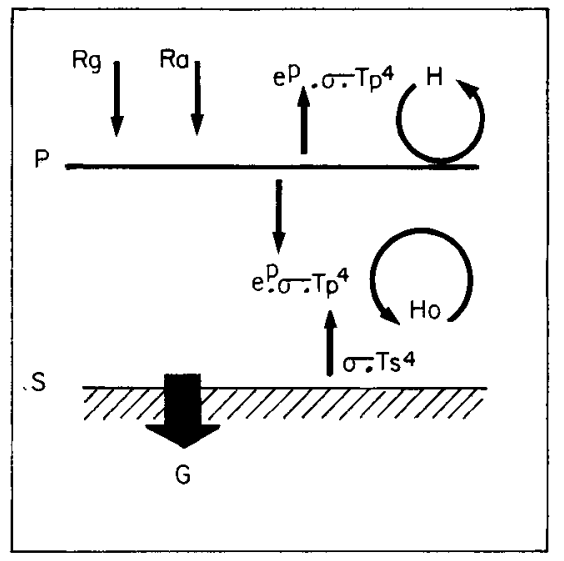

Figure 1

Représentation schématique des échanges.

Diagram of the model.

$H$ : Flux de chaleur sensible entre le paillage et l'air ambiant. Heat flux between mulch and air.

$H_{0}$ : Flux de chaleur sensible entre le paillage et la surface du sol, fonction de la résistance de contact $r_{c}$ (formule 3). Heat flux between mulch and soil (function of the contact resistance $r_{c}$ formule 3).

$\sigma$ : Constante de Stefan-Boltzmann, Stefan-Boltzmann constant.

$R a$ : Rayonnement atmosphérique de grande longueur d'onde. Longwave sky radiation.

$R g$ : Rayonnement solaire global. Solar radiation.

Ts : Température du sol $\left({ }^{\circ} \mathrm{K}\right)$. Soil temperature $\left({ }^{\circ} \mathrm{K}\right)$.

$T p$ : Température du paillage $\left({ }^{\circ} \mathrm{K}\right)$. Mulch temperature $\left({ }^{\circ} \mathrm{K}\right)$

$e^{p}:$ Emissivité du paillage. Mulch emissivity.

$G$ : Flux de chaleur par conduction dans le sol. Soil heat flux.

Pour ce travail préliminaire d'évaluation agronomique des potentialités d'échauffement du sol à partir de paillages artificiels, nous avons choisi le premier type de modèle. Les résultats obtenus montreront en effet que, malgré sa simplicité, il permet d'analyser correctement les modifications microclimatiques engendrées par la présence du paillage et leurs conséquences sur les températures du sol. L'étude présentée ici est basée sur des comparaisons de simulations et de résultats expérimentaux. Elle sera poursuivie ultérieurement par un calage rigoureux de ce modèle, une étude complète de sensibilité aux différents facteurs et une évaluation du poids des différentes hypothèses simplificatrices.

Les transferts thermiques dans un sol sont décrits par l'équation de diffusion de la chaleur qui, dans le cas unidimensionnel, s'écrit :

$$
\mathrm{dT} / \mathrm{dt}=\mathrm{a} \cdot \mathrm{d}^{2} \mathrm{~T} / \mathrm{dz} \mathrm{z}^{2}
$$

où $T$ est la température du sol, $t$ le temps, $z$ la profondeur, et a la diffusivité thermique du sol.

La condition à la limite inférieure est une condition de flux nul qui se traduit par une température constante à une profondeur fixée à $1 \mathrm{~m}$. En ce qui concerne la surface du sol, la condition à la limite est double car elle doit déterminer la température de surface du sol et la température du paillage ; cette double condition s'exprime par les bilans d'énergie de la surface du sol et du paillage :

\section{Pour le sol}

$$
\begin{aligned}
& \mathrm{G}=\mathrm{t}_{\mathrm{p}} \cdot\left(1-\mathrm{r}_{\mathrm{s}}\right) \cdot \mathrm{Rg}+\mathrm{t}^{\mathrm{p}} \cdot \mathrm{Ra}+\mathrm{e}^{\mathrm{p}} \cdot \sigma \cdot \mathrm{Tp}^{4}- \\
& \sigma . \mathrm{Ts}^{4}+\mathrm{H}_{0}
\end{aligned}
$$

où $\mathrm{G}$ est le flux de chaleur par conduction dans le sol, $\mathrm{Rg}$ le rayonnement solaire global, $\mathrm{Ra}$ le rayonnement atmosphérique, $\mathrm{Tp}$ la température du paillage, Ts la température de surface du sol. Les coefficients $t, r$, e sont les facteurs de transmission, de réflexion et les émissivités ; les indices $\mathrm{p}$ et $\mathrm{s}$ se rapportent respectivement au paillage et au sol, un indice inférieur indique qu'il s'agit de la propriété radiative dans le domaine spectral de courte longueur d'onde alors qu'un indice supérieur concerne le domaine de grande longueur d'onde. $\sigma$ est la constante de StefanBoltzmann.

$\mathrm{H}_{0}$ est le flux de chaleur sensible entre le paillage et la surface du sol. L'expression exacte de $\mathrm{H}_{0}$ n'est pas connue car elle dépend de la nature du contact entre le paillage et la surface du sol, qui varie d'un point à l'autre. Dans son modèle, MAHRER (1979) utilise une expression proposée par KimbaLL (1973) pour le flux de chaleur sensible à l'intérieur d'une serre entre le sol et la toiture. L'utilisation de la notion de résistance de contact nous a semblé préférable de par sa simplicité : elle permet d'éviter une description complexe du phénomène tout en conduisant à des résultats satisfaisants. L'expression de $\mathrm{H}_{0}$ est donc :

$$
\mathrm{H}_{0}=(1 / \mathrm{rc}) \cdot(\mathrm{Tp}-\mathrm{Ts})
$$

où rc est la résistance de contact. Généralement, rc sera prise égale à $0,04{ }^{\circ} \mathrm{C} \cdot \mathrm{m}^{-2} \cdot \mathrm{W}^{-1}$, ce qui correspond à la présence d'une couche d'air immobile d' $1 \mathrm{~mm}$ d'épaisseur entre le paillage et la surface du sol (DE VRIES, 1963).

\section{Pour le paillage}

Le bilan d'énergie s'écrit :

$$
\begin{aligned}
& \left(1-t_{p}\right) \cdot\left(1+r_{s} \cdot t_{p}\right) \cdot R g+\left(1-t^{p}\right) \cdot R a-\quad(4) \\
& \quad+2 e^{p} \cdot \sigma \cdot T^{4}+\left(1-t^{p}\right) \cdot \sigma \cdot T s^{4}+H-H_{0}=0
\end{aligned}
$$

$\mathrm{H}$ est le flux de chaleur sensible échangée par convection au niveau de la face supérieure du paillage, qui peut s'exprimer sous la forme (ANTONIOLETTI \& BAILLE, 1982) :

$$
\mathrm{H}=\mathrm{hc} \cdot(\mathrm{Ta}-\mathrm{Tp})
$$

où Ta est la température de l'air et hc un coefficient d'échange qui peut s'exprimer simplement (sans correction de stabilité thermique de l'air) en fonction de la 
vitesse de l'air à $2 \mathrm{~m}, \mathrm{~V}$, d'après Mac ADAMS, 1954 (cité par ANTONIOLETTI \& BAILlE, 1982) :

$$
\mathrm{hc}=3,7 . \mathrm{V}+5,6\left(\mathrm{hc} \text { en } \mathrm{W} \cdot \mathrm{m}^{-2} \cdot{ }^{\circ} \mathrm{C}^{-1}, \mathrm{~V} \text { en } \mathrm{m} \cdot \mathrm{s}^{-1}\right)
$$

Le champ de température du sol dépend non seulement des propriétés thermophysiques du sol et du paillage mais aussi des variables climatiques. Une évolution standard du rayonnement global, établie dans nos conditions, a été choisie ; elle permet de simuler la variation journalière à partir de la seule mesure du rayonnement solaire journalier. Compte tenu de la très faible variabilité des températures moyennes journalières, une évolution sinusoïdale autour de $24{ }^{\circ} \mathrm{C}$ a été utilisée. Le rayonnement atmosphérique peut être supposé constant (CANDELON, 1986) et des vitesses de vent de jour doubles de celles de nuit reflètent assez bien nos conditions d'alizés. Ces valeurs standards (tabl. 1) permettent d'évaluer simplement les variables climatiques qui ne sont pas disponibles en permanence.

TABLEAU 1

Evolution standard des variables climatiques simulées. Standard time-course of the simulated climatic parameters.

RAYONNEMENT GLOBAL $\left(W . \mathrm{m}^{-2}\right.$ ) d'après SINOQUET (1985)

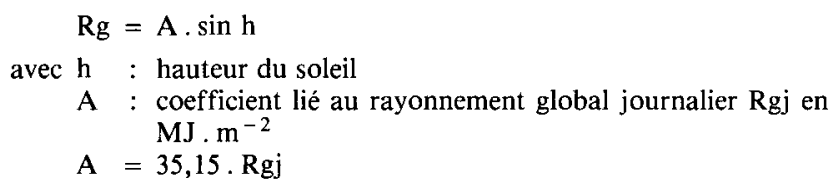

RAYONNEMENT ATMOSPHÉRIQUE (W . $\mathrm{m}^{-2}$ )

$\mathrm{Ra}=400$

TEMPÉRATURE DE L'AIR

$$
\begin{aligned}
& \text { Ta }=24+3,5 \cdot \sin (2 \pi / 24(\mathrm{t}-8)) \\
& \text { avec } \mathrm{t} \quad: \text { heure de la journée } \\
& \text { VITESSE DE L'AIR À } 2 \mathrm{~m}\left(\mathrm{~m} \cdot \mathrm{s}^{-1}\right) \\
& \qquad \begin{aligned}
\mathrm{V} & =3 \text { de jour }(6 \mathrm{~h}<\mathrm{t}<18 \mathrm{~h}) \\
\mathrm{V} & =1,5 \text { de nuit }(18 \mathrm{~h}<\mathrm{t}<6 \mathrm{~h})
\end{aligned}
\end{aligned}
$$

La résolution numérique des équations est effectuée grâce à un schéma explicite de différences finies, avec un pas d'espace de $1 \mathrm{~cm}$ et un pas de temps de $1 \mathrm{mn}$. Des solutions numériques plus optimales seront recherchées ultérieurement.

Cette approche des phénomènes thermiques dans le sol paillé est simplifiée, notamment au niveau des bilans radiatifs où le sol est assimilé à un corps noir et les coefficients de réflexion du paillage sont supposés nuls. De plus, le paillage est supposé stopper toute évaporation du sol et les phénomènes de condensation ou de transfert de chaleur latente entre la surface du sol et le paillage ne sont pas pris en compte.

\section{B. Dispositif expérimental}

Les expériences se sont déroulées à la Guadeloupe, au Domaine Duclos du Centre I.N.R.A. des Antilles $\left(16^{\circ} 15^{\prime} \mathrm{N}, 61^{\circ} 40^{\prime} \mathrm{W}\right)$.

\section{Caractéristiques des parcelles}

Les parcelles, de dimension $4 \times 7 \mathrm{~m}$, sont situées dans une zone de sol d'alluvions ferrallitisés. Ce sol est composé de 7 p. 100 de sable grossier, 7 p. 100 de sable fin, 6 p. 100 de limon grossier, 23 p. 100 de limon fin, et 57 p. 100 d'argile (halloysite). La densité apparente sèche est environ de $0,9 \mathrm{~g} . \mathrm{cm}^{-3}$; la teneur en eau moyenne est égale à $0,4 \mathrm{~g} \cdot \mathrm{g}^{-1}$ et atteint $0,6 \mathrm{~g} \cdot \mathrm{g}^{-1}$ à la capacité au champ. Les parcelles ont été désherbées chimiquement avant l'application des paillages et ont été entretenues durant toute la campagne de mesure par désherbage manuel régulier.

\section{Essai $n^{\circ} 1$ : Comparaison entre différents types de paillage}

Les mesures ont eu lieu durant le mois d'avril 1985. Trois types de paillage ont été utilisés. Il s'agissait de plaques de verre de dimension $1 \times 2 \mathrm{~m}$ et d'épaisseur $3 \mathrm{~mm}$, d'un film plastique transparent en polyéthylène basse-densité d'épaisseur 90 microns, et d'un film plastique opaque noir en polyéthylène d'épaisseur $30 \mathrm{mi}$ crons (type « spécial fraise »). Ces matériaux ont été choisis en raison de leurs propriétés optiques différentes. Bien que l'utilisation au champ de plaques de verre soit difficilement réalisable, ce matériau a été également testé car il est relativement transparent au rayonnement de petite longueur d'onde et opaque au rayonnement infrarouge. Le film plastique transparent a, quant à lui, une bien meilleure transmission dans ce dernier domaine, et le film plastique noir est pratiquement opaque dans les deux gammes spectrales (tabl. 2). Les paillages ont été appliqués sur un sol dont l'état hydrique n'a pas été modifié, et donc non saturé en eau.

\section{TABLEAU 2}

Propriétés radiatives des paillages utilisés (D'après Liakatas et al. (1986) et NiSEN (1979)). Radiative properties of mulches.

\begin{tabular}{lccccccc}
\hline & \multicolumn{5}{c}{ Domaine de longueurs d'onde } \\
\cline { 2 - 8 } & \multicolumn{4}{c}{$400-2500 \mathrm{~nm}$} & \multicolumn{4}{c}{$5-35 \mu \mathrm{m}$} \\
\hline Paillage & $\mathrm{t}_{\mathrm{p}}$ & $\mathrm{r}_{\mathrm{p}}$ & $\mathrm{a}_{\mathrm{p}}$ & $\mathrm{t}_{\mathrm{p}}$ & $\mathrm{r}_{\mathrm{p}}$ & $\mathrm{a}_{\mathrm{p}}$ \\
$\begin{array}{l}\text { Verre } \\
\text { Film plastique opaque }\end{array}$ & 0,80 & 0,15 & 0,05 & 0,00 & 0,00 & 1,00 \\
noir & 0,00 & 0,10 & 0,90 & 0,15 & 0,00 & 0,85 \\
Film plastique transpa- \\
rent
\end{tabular}

$\left(\mathrm{t}_{\mathrm{p}}\right.$ : facteur de transmission ; transmissivity),

$\left(\mathrm{r}_{\mathrm{p}}\right.$ : facteur de réflexion; reflectivity),

$\left(a_{\mathrm{p}}\right.$ : facteur d'absorption; absorptivity).

La température a été mesurée à $2 \mathrm{~cm}$ de profondeur (avec une erreur de localisation d'environ $+/-0,25 \mathrm{~cm}$ ) par des thermocouples cuivre-constantan. Les soudures chaudes étaient ancrées dans le sol grâce à une armature métallique, de manière à éviter un déplacement du capteur au cours du temps. La soudure froide était placée dans le sol, à $50 \mathrm{~cm}$ de profondeur, où la température était constante pendant la durée de l'essai. Pour chaque parcelle, la température était mesurée en 6 points par deux groupes de thermocouples montés en série. Des mesures ont été également effectuées sur une parcelle témoin non paillée. 


\section{Essai $n^{\circ} 2$ : Pénétration de l'onde thermique}

Cet essai a été mené sur les mêmes parcelles durant le mois de décembre 1985. Le verre a été choisi comme matériau de paillage car il permettait d'obtenir, de par ses propriétés optiques, un écart maximal de température entre la parcelle paillée et la parcelle non paillée. Les températures ont été mesurées aux profondeurs $2,5 \mathrm{~cm}, 5 \mathrm{~cm}, 7,5 \mathrm{~cm}, 15 \mathrm{~cm}$ et $25 \mathrm{~cm}$ grâce à une série de thermocouples. Les fils de soudure chaude étaient tendus sur un bâti en forme de "lyre ", permettant de maintenir les capteurs à la cote désirée. Les cadres ont été placés dans le sol en prenant soin de modifier le moins possible sa structure ; à cet effet, les fosses ont été creusées et rebouchées couche par couche. Les parcelles ont été arrosées jusqu'à saturation avant l'application du paillage, et la parcelle témoin (non paillée) était maintenue à saturation par arrosage régulier, de manière à maintenir des conditions hydriques similaires sur chaque parcelle.

\section{Traitement des données}

Les thermocouples étaient reliés à une centrale de mesure CR21 Micrologger (Campbell Scientific Inc.), scrutant les capteurs chaque minute et réalisant des moyennes horaires. La mémoire de la centrale permettant de stocker $48 \mathrm{~h}$ de mesures était régulièrement copiée et son contenu transféré sur un microordinateur Apple II, grâce à un programme d'interface (VALMORIN, 1986).

\section{RÉSULTATS}

\section{A. Validation du modèle}

La validation du modèle a consisté essentiellement à comparer les températures maximales journalières mesurées, à celles issues de la modélisation. En effet, les températures maximales nous semblent des critères très importants pour caractériser l'efficacité du paillage dans un but de désinfection. Seules les mesures du second essai ont été utilisées car les propriétés thermophysiques du système sol-paillage étaient bien définies ; la diffusivité thermique du sol ayant été mesurée et sa capacité calorifique déterminée à partir de la répartition volumique de ses constituants à la capacité au champ (DE VRIES, 1963 ; tabl. 3).

L'écart moyen entre températures maximales journalières mesurées et calculées, défini par la moyenne des valeurs absolues des différences de ces valeurs, est de l'ordre de $1,3{ }^{\circ} \mathrm{C}$. MAHRER (1979) indiquait, pour son modèle et pour l'ensemble des températures horaires, un écart moyen variant de 0,6 à $1,3^{\circ} \mathrm{C}$ en fonction de l'état hydrique du sol et de la profondeur considérée. La figure 2 montre cependant que le modèle est légèrement biaisé : les températures maximales sont surestimées pour les faibles profondeurs et les fortes valeurs de rayonnement, alors qu'elles sont sousestimées pour les profondeurs plus importantes et pour les faibles valeurs de rayonnement. Ceci peut s'expliquer par la conjonction de deux faits : d'une part, la diffusivité thermique du sol a pu être sous-estimée, provoquant ainsi, lors de la simulation, des écarts trop importants des températures maximales entre les faibles profondeurs $(2,5 \mathrm{~cm})$ et les couches plus profondes $(15$ et $25 \mathrm{~cm})$. D'autre part, la surestimation de

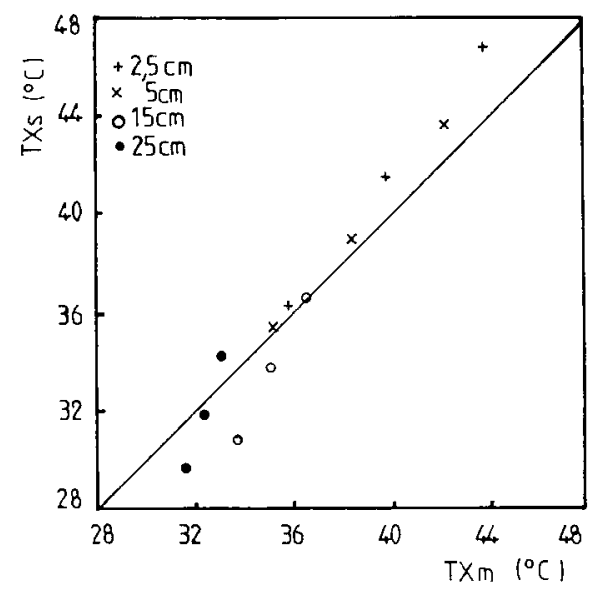

Figure 2

Comparaison entre les températures maximales journalières mesurées à différentes profondeurs (TXm) et calculées par simulation (TXS) sous la plaque de verre.

Comparison between the maximum daily temperatures measured at several depths (TXm) and simulated (TXs) under glass.

l'efficacité du paillage pour les rayonnements élevés pose le problème de la détermination des propriétés radiatives des matériaux au champ, qui peuvent être différentes de celles obtenues en laboratoire ou évoluer au cours du temps en fonction de l'âge du paillage (AviSSAR et al., 1986). Il est à noter qu'une variation de 0,8 à 0,7 de la transmissivité du verre dans le domaine des petites longueurs d'onde réduit l'écart moyen de $0,1{ }^{\circ} \mathrm{C}$.

TABLEAU 3

Caractéristiques thermiques du sol en fonction de son état hydrique.

Thermal characteristics of soil with respect to soil water content.

\begin{tabular}{lccc}
\hline \hline \multicolumn{1}{c}{ Etat du sol } & Capacité au champ & Etat moyen & Etat sec \\
\hline Fraction volumique d'eau & 0,65 & 0,45 & 0,30 \\
Diffusivité thermique $\left(\mathrm{m}^{2} \cdot \mathrm{s}^{-1}\right)$ & $2,75 \cdot 10^{-7}(1)$ & $3,30 \cdot 10^{-7}(2)$ & $3,70.10^{-7}(2)$ \\
Capacité calorifique volumique $\left(\mathrm{J}^{\prime} \mathrm{m}^{-3} \cdot{ }^{\circ} \mathrm{K}^{-1}\right)(3)$ & $3,50.10^{6}$ & $2,50 \cdot 10^{6}$ & $1,90.10^{6}$ \\
Conductivité thermique $\left(\mathrm{W} . \mathrm{m}^{-1} \cdot{ }^{\circ} \mathrm{K}^{-1}\right)$ & 0,95 & 0,82 & 0,70 \\
\hline \hline
\end{tabular}

(1) Mesurée (méthode de KIRKHAM \& PowERS, 1972, basée sur l'atténuation de l'amplitude thermique en fonction de la profondeur).

(2) Déduite graphiquement des courbes de BAVER et al., 1972

(3) Calculée (méthode de DE VRIES, 1963). 


\section{B. Comparaison des différents paillages}

La figure 3 montre l'évolution de la température du sol à $2 \mathrm{~cm}$ sous les parcelles paillées et la parcelle non paillée sur une période de 2 jours. Le paillage le plus efficace pour le chauffage du sol est le verre, suivi du film plastique transparent puis du film plastique opaque noir. Pour un rayonnement global moyen de $17,5 \mathrm{MJ} \mathrm{.} \mathrm{m}^{-2}$. jour ${ }^{-1}$, la température maximale journalière était égale à $54{ }^{\circ} \mathrm{C}$ pour le sol recouvert de plaques de verre, $51{ }^{\circ} \mathrm{C}$ pour le plastique transparent, $45^{\circ} \mathrm{C}$ pour le plastique opaque noir et $38{ }^{\circ} \mathrm{C}$ pour le sol non paillé. La présence du paillage a donc entraîné une augmentation de la température maximale de 7 à $16{ }^{\circ} \mathrm{C}$, selon le paillage.

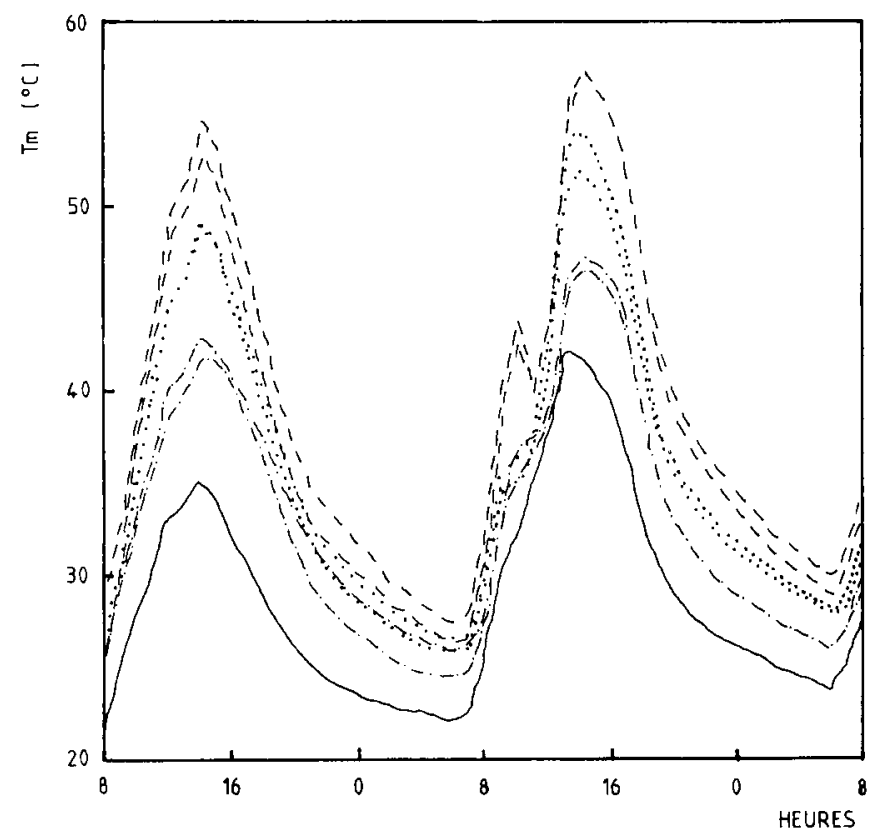

Figure 3

Evolution de la température du sol mesurée à $2 \mathrm{~cm}(T \mathrm{Tm})$ sous différents paillages.

Measured soil temperature at $2 \mathrm{~cm}$ depth (Tm) under several mulches.

.... Plaque de verre (glass)

.... Plastique transparent (clear plastic film)

-.- Plastique opaque noir (black plastic film)

- Sol nu (bare soil)
La température maximale journalière est bien corrélée au rayonnement solaire global journalier (tabl. 4). Ces résultats sont cohérents puisque l'augmentation de température due à la présence du paillage dépend de deux phénomènes principaux fonction du rayonnement solaire incident : d'une part, le paillage modifie le bilan radiatif de surface; d'autre part, il supprime l'évaporation du sol. Cependant, les températures maximales sont liées plus fortement au rayonnement global journalier, que ne le sont les écarts de température maximale provoqués par la présence du paillage. Quant aux températures minimales journalières, leur degré de dépendance avec le rayonnement solaire est faible ; elles sont par contre liées aux températures maximales atteintes la veille.

\section{TABLEAU 4}

Coefficients de corrélation entre le rayonnement solaire journalier $\mathrm{Rg}$ et les températures à $2 \mathrm{~cm}$ pour les sols nus et paillés. $N=18$.

Correlation coefficients between daily solar radiation $\mathrm{Rg}$ and temperature at a depth of $2 \mathrm{~cm}$ for bare and mulched soils. $N=18$

\begin{tabular}{lcccc}
\hline \hline & $\mathrm{Rg} / \mathrm{TX}_{\mathrm{j}}$ & $\mathrm{Rg} / \mathrm{DTX}_{\mathrm{j}}$ & $\mathrm{Rg} / \mathrm{TN}_{\mathrm{j}}$ & $\mathrm{TX}_{\mathrm{j}-1} / \mathrm{TN}_{\mathrm{j}}$ \\
\hline Verre & 0,888 & 0,768 & 0,526 & 0,690 \\
Plastique transparent & 0,827 & 0,540 & 0,100 & 0,680 \\
Plastique opaque noir & 0,827 & 0,560 & 0,090 & 0,650 \\
Sol nu & 0,748 & - & - & 0,400 \\
\hline \hline
\end{tabular}

$\mathrm{TX}_{\mathrm{j}}$ : Température maximale journalière. Daily maximum temperature.

DTX $_{\mathrm{j}}$ : Ecart de température maximale journalière entre sol paillé et sol nu. Daily maximum temperature difference between mulched and bare soil.

$\mathrm{TN}_{\mathrm{j}} \quad$ : Température minimale journalière. Daily minimum temperature.

\section{Pénétration de l'onde thermique}

La figure 4 montre l'évolution, sur une période de 2 jours, des écarts de température entre le sol recouvert de plaques de verre et le sol non paillé. Ces écarts sont particulièrement importants pour les températures maximales journalières. Ils diminuent en fonction de la profondeur : à $2,5 \mathrm{~cm}$, l'écart moyen sur les températures maximales journalières est égal à $10,2{ }^{\circ} \mathrm{C}$; il atteint $8,9^{\circ} \mathrm{C}$ à $5 \mathrm{~cm}, 8,2{ }^{\circ} \mathrm{C}$ à $7,5 \mathrm{~cm}, 6,7{ }^{\circ} \mathrm{C}$ à $15 \mathrm{~cm}$ et $5,5^{\circ} \mathrm{C}$ à $25 \mathrm{~cm}$.

Measured temperature differences (DTm) between mulched soil (with glass) and bare soil for various depths.

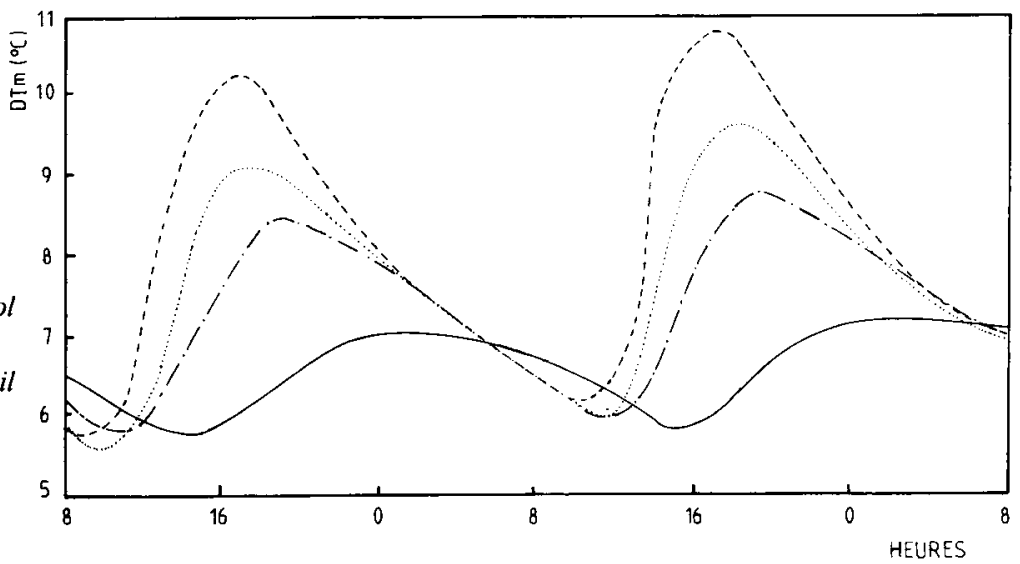


Les écarts de température maximaux créés par le verre sont liés au rayonnement solaire journalier mais le coefficient de corrélation diminue lorsque la profondeur augmente (tabl. 5). Il en est de même pour la pente de la droite de régression liant écarts de températures et rayonnement solaire; le terme constant est quant à lui peu variable.

\section{TABLEAU 5}

Relations entre le rayonnement solaire journalier ( $\mathrm{Rg}$ en $\left.\mathrm{MJ}, \mathrm{m}^{-2}\right)$ et les écarts maximaux journaliers de température sol avec verre-sol $n u\left(D T X\right.$ en $\left.{ }^{\circ} \mathrm{C}\right)$, à différentes profondeurs.

Relation between daily solar radiation ( $\mathrm{Rg}$ in $\left.\mathrm{MJ} . \mathrm{m}^{-2}\right)$ and daily maximal differences (DTX in ${ }^{\circ} \mathrm{C}$ ) between mulched (with glass) and non-mulched soils, at different depths.

\begin{tabular}{cccc}
\hline \hline Profondeur & \multicolumn{3}{c}{ Relation } \\
\hline $2,5 \mathrm{~cm}$ & DTX $=0,512 \mathrm{Rg}+2,952$ & $\mathrm{r}^{2}=0,894$ & $\mathrm{~N}=18$ \\
$5,0 \mathrm{~cm}$ & DTX $=0,380 \mathrm{Rg}+3,441$ & $\mathrm{r}^{2}=0,849$ & $\mathrm{~N}=11$ \\
$7,5 \mathrm{~cm}$ & DTX $=0,363 \mathrm{Rg}+3,082$ & $\mathrm{r}^{2}=0,807$ & $\mathrm{~N}=18$ \\
$15,0 \mathrm{~cm}$ & DTX $=0,253 \mathrm{Rg}+3,120$ & $\mathrm{r}^{2}=0,685$ & $\mathrm{~N}=18$ \\
\hline
\end{tabular}

\section{DISCUSSION}

\section{A. Comparaison des différents paillages}

Pour comparer les paillages, les températures maximales quotidiennes mesurées ont été ajustées au rayonnement global journalier par régression linéaire (fig. 5). La dispersion des points est assez forte dans le cas du sol nu, mais elle reste sensible avec les paillages, sans doute à cause de l'influence du passé thermique d'un jour sur l'autre. Ces droites traduisent l'efficacité des paillages, et les caractéristiques radiatives des matériaux devraient permettre, à l'aide du modèle, de

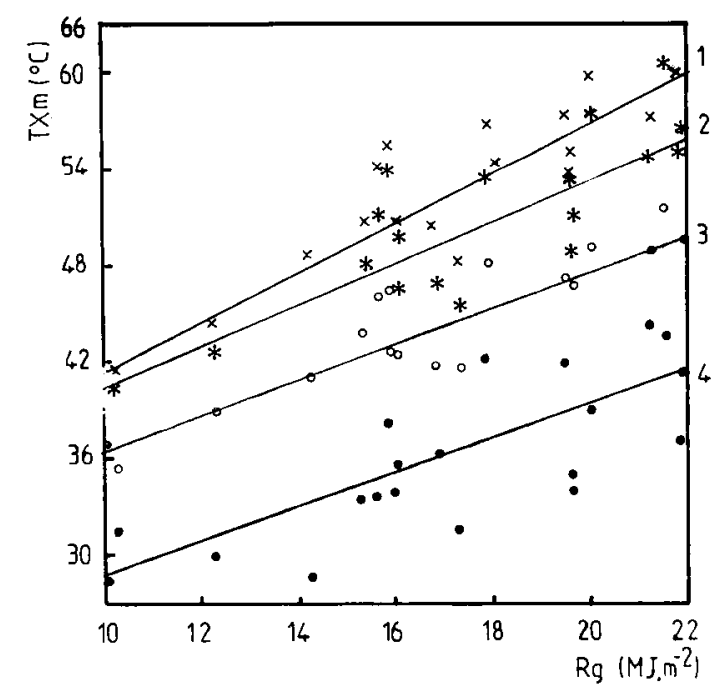

Figure 5

Relations entre la température maximale journalière mesurée à $2 \mathrm{~cm}$ sous différents paillages (TXm) et le rayonnement global journalier (Rg).

Relationship between maximum daily temperature at $2 \mathrm{~cm}$ depth under various mulches $(T X m)$ and daily solar radiation $(R g)$.

$1: \times$ Verre (glass)

2: * Plastique transparent (clear plastic)

3: O Plastique opaque noir (black plastic)

4:- Sol nu (bare soil) quantifier les modifications microclimatiques engendrées par la présence du paillage.

Dans le cas du verre et du film plastique transparent, les températures maximales simulées sont plus élevées que celles obtenues pour un sol nu sans évaporation (correspondant à un paillage dont les propriétés seraient : $t_{p}=t^{p}=1$ et $\left.e^{p}=0\right)$; par contre, elles sont inférieures dans le sol recouvert par le film plastique opaque noir (fig. 6).

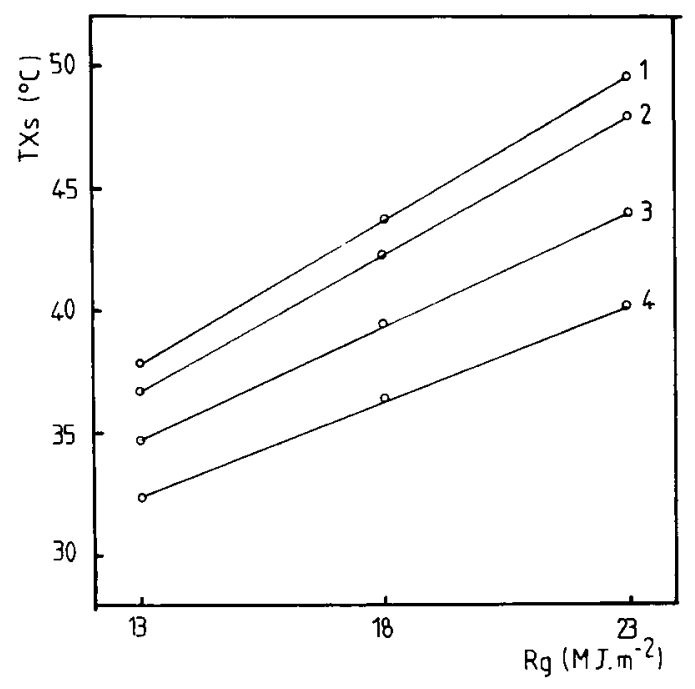

Figure 6

Influence du rayonnement global journalier (Rg) sur la température maximale journalière simulée (TXS) à $2 \mathrm{~cm}$ de profondeur sous différents paillages et dans un sol nu sans évaporation, avec un état hydrique moyen.

Influence of daily solar radiation $(R g)$ on daily maximum temperature simulated (TXs) at a $2 \mathrm{~cm}$ depth under different mulches and bare soil without evaporation, with intermediate-soil water content.

Pentes (slopes)

I: Verre (glass)

1,16

2 : Plastique transparent (clear plastic)

1,10

3: Sol nu sans évaporation (bare soil without evaporation)

0,92

4 : Plastique opaque noir (black plastic)

0,76

\section{Cas des paillages transparents}

A la suppression de l'évaporation s'ajoute, pour les matériaux transparents, une augmentation du flux de chaleur sensible absorbé par le sol. Ce gain d'énergie ne paraît pas être d'origine radiative puisque les rayonnements absorbés par le sol nu sans évaporation et le sol recouvert de plaques de verre sont du même ordre de grandeur (fig. 7) ; il n'est supérieur, dans le cas du verre, que la nuit où l'effet de serre se fait sentir. Dans la journée, la réduction des pertes radiatives de grande longueur d'onde compense à peine la diminution d'absorption de rayonnement solaire dû au coefficient de transmission du paillage. Le rayonnement absorbé par le sol paillé avec le film plastique transparent est, durant la journée, plus faible que dans le cas du sol nu sans évaporation et la nuit, du même ordre de grandeur ; ceci est conforme aux mesures de rayonnement net effectuées sous le paillage par LIAKATAS et al. (1986). La diminution des pertes sensibles semble donc avoir pour cause une réduction des échanges convectifs entre la surface du sol et l'air ambiant, due à la lame d'air emprisonnée sous le paillage et jouant un rôle 


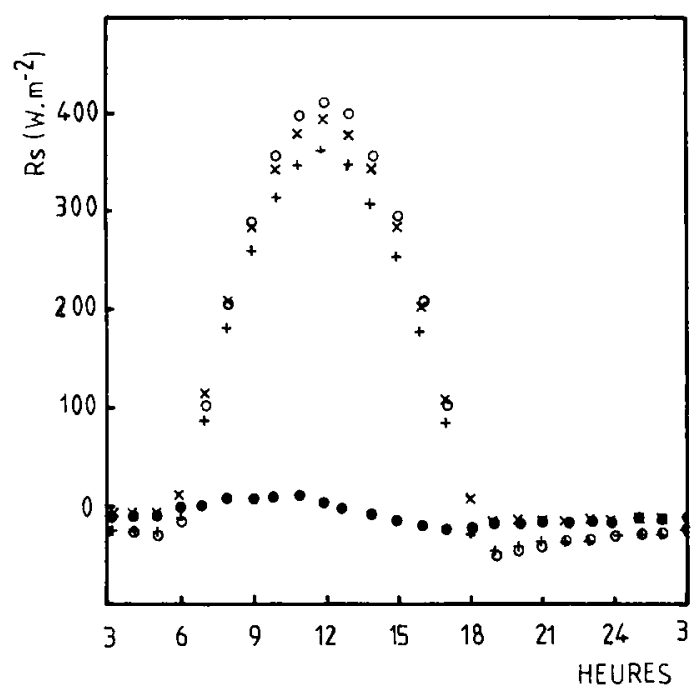

Figure 7

Simulation du rayonnement absorbé par le sol (Rs) sous différents paillages et par le sol nu sans évaporation au cours d'une journée standard avec un rayonnement global journalier de $18 \mathrm{MJ} . \mathrm{m}^{-2}$ et un état hydrique moyen du sol.

Simulation of the absorbed radiation in the soil (Rs) under different mulches, and also by a bare soil without evaporation, during a standard day with a daily global radiation $R g=18 \mathrm{MJ} . \mathrm{m}^{-2}$ and $a$ medium soil water content.

$x:$ Verre (glass)

* : Plastique transparent (clear plastic)

- : Plastique opaque noir (black plastic)

0 : Sol nu sans évaporation (bare soil without evaporation)

d'isolant. Les températures maximales sont d'ailleurs d'autant plus élevées que la résistance de contact rc, qui caractérise l'isolation de la couche d'air, est plus grande (fig. 8) ; dans le cas extrême où rc est nulle, les températures de surface du sol et du paillage sont identiques et la condition limite devient :

$$
\mathrm{G}=\left(1-\mathrm{r}_{\mathrm{s}} \cdot \mathrm{t}_{\mathrm{p}}{ }^{2}\right) \cdot \mathrm{Rg}+\mathrm{Ra}-\sigma \cdot \mathrm{Ts}^{4}+\mathrm{H}
$$

alors qu'elle s'écrit, dans le cas du sol nu sans évaporation :

$$
\mathrm{G}=\left(1-\mathrm{r}_{\mathrm{s}}\right) \cdot \mathrm{Rg}+\mathrm{Ra}-\sigma \cdot \mathrm{Ts}^{4}+\mathrm{H}
$$

Le flux de chaleur absorbé par le sol paillé reste donc supérieur à celui du sol nu sans évaporation, mais les différences s'estompent pour les coefficients de transmission élevés dans le domaine des courtes longueurs d'onde.

\section{Cas du paillage opaque}

Le rayonnement absorbé par le sol est pratiquement nul tout au long de la journée ; il est légèrement positif seulement le matin (fig. 7). L'apport diurne de chaleur sensible n'est pas d'origine radiative ; il est dû aux échanges conductifs, voire convectifs, qui ont lieu entre le paillage et la surface du sol, la température du paillage étant supérieure à celle de la surface du sol. Cet apport est d'autant plus important que l'isolation de la lame d'air est plus faible : ceci se traduit par une augmentation des températures maximales lorsque la résistance de contact sol-paillage diminue (fig. 8). Le mode de chauffage du sol est donc différent du cas des matériaux transparents puisque le rayonnement solaire est absorbé par le paillage et l'énergie ainsi stockée redistribuée au sol par conduction. A ce sujet, LIAKA-

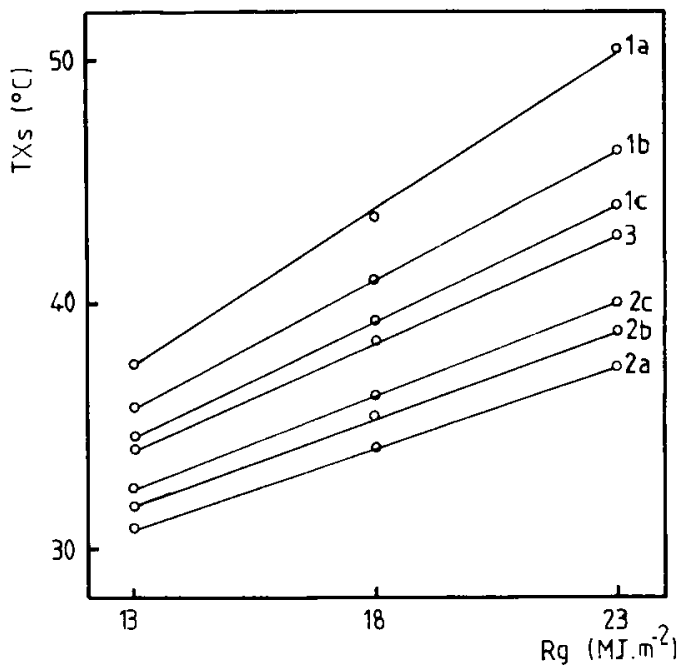

Figure 8

Simulation de l'influence de la résistance de contact paillage-sol $(r d)$ sur la relation entre température maximale journalière d $2 \mathrm{~cm}$ (TXs) et le rayonnement global journalier $(R g)$. Sol nu sans évaporation et différents paillages; sol à la capacité au champ.

Simulation of the influence of the contact resistance mulch-soil $\left(r_{d}\right)$ on the relationship between maximum daily temperature at $2 \mathrm{~cm}$ depth (TXs) and daily solar radiation (Rg). Bare soil without evaporation and differents mulches; soil water content at field capacity.

Pentes (slopes)

$\begin{array}{lr}\text { 1: Plastique transparent (clear plastic) } & \\ a: r_{c}=0,08{ }^{\circ} \mathrm{C} \cdot \mathrm{m}^{-2} \cdot W^{-1} & 1,18 \\ b: r_{c}=0,04 & 1,04 \\ c: r_{c}=0,02 & 0,92 \\ 2: \text { Plastique opaque noir (black plastic) } & \\ a: r_{c}=0,08 & 0,63 \\ b: r_{c}=0,04 & 0,70 \\ c: r_{c}=0,02 & 0,75 \\ 3: \text { Sol nu sans évaporation (bare soil without evaporation) } & 0,86\end{array}$

TAS et al. (1986) indiquent qu'un paillage plastique opaque n'est efficace que s'il est en contact intime avec le sol.

En ce qui concerne les matériaux transparents, le gradient thermique dans la lame d'air est tel qu'il favorise le développement d'instabilités thermoconvectives qui accroissent le transfert de chaleur sensible entre la surface du sol et le paillage. Par contre, pour le film opaque, le gradient de température dans la couche d'air est inversé, ce qui a pour effet de limiter les échanges convectifs entre les deux surfaces. Dans les deux cas, et dans un but de chauffage du sol, l'état de stabilité thermique de la lame d'air joue dans le mauvais sens puisqu'il diminue l'isolation de la couche d'air pour le verre et le film plastique transparent et oppose une résistance au transfert de chaleur du paillage vers le sol dans le cas du film plastique opaque noir.

\section{B. Profils de température et état hydrique du sol}

L'augmentation de température maximale liée à l'action du paillage est assez différente d'un essai sur l'autre ; en effet, dans le premier essai, pour un rayonnement solaire de $20 \mathrm{MJ} . \mathrm{m}^{-2}$. jour ${ }^{-1}$, l'écart de température maximale observé par rapport au sol nu à la profondeur $2 \mathrm{~cm}$ sous le verre est de l'ordre de $18^{\circ} \mathrm{C}$ alors qu'il n'atteint que $13{ }^{\circ} \mathrm{C}$ dans le second essai pour le même paillage, un rayonnement solaire identi- 
que et une profondeur équivalente $(2,5 \mathrm{~cm})$. Ces différences peuvent être dues à l'état hydrique du sol puisqu'il n'était saturé que dans le second essai ; cette situation joue sur les propriétés thermiques du sol dans le sens d'une augmentation de la conductivité thermique et de la capacité calorifique (DE VRIES, 1963) ; elle devrait donc favoriser la pénétration de la chaleur en profondeur mais réduire les températures puisque le stockage d'une même quantité de chaleur provoque une élévation de température moindre.

Lors de la simulation, 3 états hydriques ont été comparés : capacité au champ, état hydrique moyen et état hydrique plutôt sec correspondant à des fractions volumiques en eau de 65 p. 100,40 p. 100 et 30 p. 100 . Les capacités calorifiques ont été déterminées par la méthode de DE VRIES (1963) et les diffusivités thermiques des états moyen et plutôt sec ont été déduites des courbes fournies par BAVER et al. (1972) et reprises par CAMPBELl (1977) (tabl. 3). Dans la gamme de variation de teneur en eau considérée, une augmentation de l'humidité du sol provoque un accroissement notable de la capacité calorifique, une faible diminution de la diffusivité et une faible augmentation de la conductivité.

Une humidité du sol élevée réduit les températures maximales dans la couche superficielle; pour un

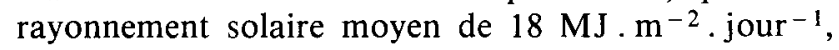
l'état hydrique plutôt sec conduit à des températures maximales simulées de $3{ }^{\circ} \mathrm{C}$ supérieures à celles calculées pour un sol à la capacité au champ (fig. 9). Cet

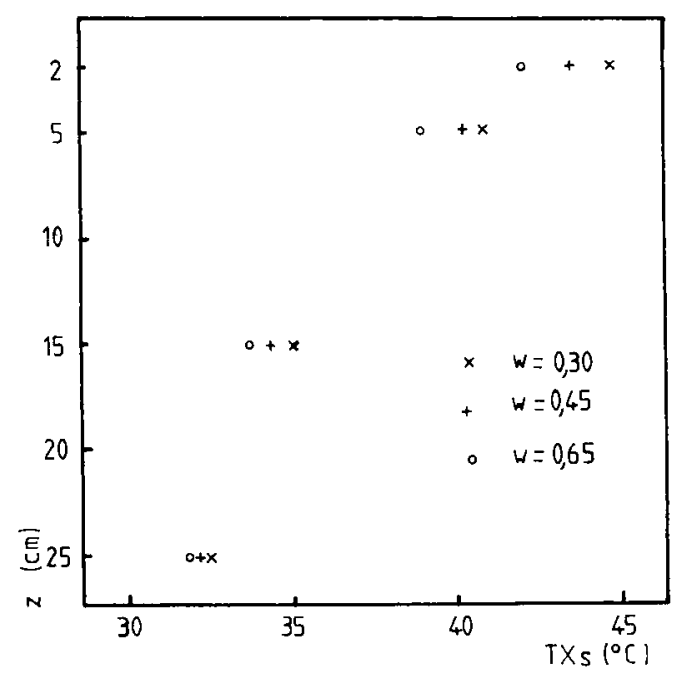

Figure 9

Simulation de l'influence de l'état hydrique du sol (caractérisé par la fraction volumique d'eau (w) sur les profils de températures maximales journalières (TXs) en fonction de la profondeur (z), avec une plaque de verre et un $\mathrm{Rg}=18 \mathrm{MJ} . \mathrm{m}^{-2}$.jour ${ }^{-1}$.

Simulation of the influence of soil water content (w) on the profile of maximum daily temperature (TXS) in relation to depth (z), with a glass cover and $\mathrm{Rg}=18 \mathrm{MJ} \cdot \mathrm{m}^{-2}$. day $\mathrm{y}^{-1}$. écart est inférieur à celui observé d'un essai sur l'autre lors des mesures, mais la différence peut s'expliquer aussi par des variations de la résistance de contact solpaillage.

L'effet de l'état hydrique du sol diminue lorsque la profondeur augmente et les différences de température maximale simulée entre sol à la capacité au champ et sol sec sont de l'ordre d' $1{ }^{\circ} \mathrm{C}$ à $25 \mathrm{~cm}$ (fig. 9). Un sol sec semble donc plus favorable pour une solarisation. Cependant, certains microorganismes du sol adoptent des formes de résistance dans les sols secs et sont ainsi capables de supporter des températures beaucoup plus importantes. D'autre part, cet effet de l'humidité du sol semble dépendre du type de sol puisque MAHRER et al. (1984) ont observé, dans le cas d'un sol argileux léger, des températures maximales plus faibles pour les fortes humidités, mais l'effet contraire dans le cas d'un sol sableux ; il est vrai que les gammes de contenu en eau de ces sols sont très différentes.

\section{CONCLUSION}

Le paillage du sol permet, à la Guadeloupe, d'obtenir un échauffement du sol assez important ; les températures maximales peuvent dépasser $50^{\circ} \mathrm{C}$ avec des variations en fonction du paillage utilisé, de l'état hydrique du sol et, évidemment, de l'intensité du rayonnement solaire et de la profondeur considérée.

En dehors de la suppression de l'évaporation, les mécanismes responsables de l'échauffement du sol paillé dépendent de la nature du paillage. En particulier, la couche d'air emprisonnée entre le paillage et la surface du sol, bien que difficile à contrôler, semble être un facteur déterminant pour l'efficacité du chauffage du sol. Quant à l'état hydrique du sol, son influence sur les températures du sol existe mais varie certainement en fonction de la nature du sol.

En ce qui concerne les Antilles, ces résultats sont encourageants et il reste maintenant à déterminer quels sont les effets de ces températures élevées sur les populations d'agents phytopathogènes. Un tel échauffement du sol devrait être a priori suffisant puisque des températures maximales de l'ordre de 45 à $50^{\circ} \mathrm{C}$ permettent une réduction notable de certaines populations de ravageurs du sol, que ce soit en Israël (KATAN et al., 1976), en Californie (JOHNSON et al., 1981) ou dans le Sud-Est de la France (BERNINGER et al., 1985). Des travaux sont en cours afin d'évaluer les aspects biologiques de la solarisation des sols aux Antilles.

Reçu le 6 janvier 1987. Accepté le 4 mai 1987.

\section{REFERENCES BIBLIOGRAPHIQUES}

Antonioletti R. \& Baille A., 1982. Effets thermiques d'un mulch pétrolier et d'un conditionneur : comparaison des résultats expérimentaux avec un modèle analytique. Note interne, I.N.R.A. STEFCE, $18 \mathrm{p}$.

Avissar R., Mahrer Y., Margules L., Katan J., 1986. Field aging of transparent polyethylene mulches : I. Photometric properties. Soil Sci. Soc. Am. J., 50, 202-205.
Baver L. D., Gardner W. H., Gardner W. R., 1972. Soils physics. Wiley, New York, $4^{e}$ éd., 498 p.

Berninger E., Pionnat J. C., Scotto La Massese C., 1985. Essai de désinfection solaire des sols dans le Sud-Est de la France. Agronomie, 5, 505-513.

Campbell G. S., 1977. An introduction to environmental biophysics. Springer-Verlag, New York. 159 p. 
Candelon C., 1986. Analyse des composantes du rayonnement net d̀ la Guadeloupe. Application à l'estimation du rayonnement net diurne. Utilisation du pyranomètre à distillation BELLANI pour l'estimation du rayonnement global. Mémoire CNEARC-ESAT, I.N.R.A. Guadeloupe, 32 p. + annexes.

Johnson A. W., Jaworski C. A., Glaze N. C., Summer D. R., Chalfant R. B., 1981. Effects of film mulch and soil pesticides on nematodes, weeds and yields of vegetable crops. J. Nematol., 13, 141-148.

Katan J., Greeberger A., Alan H., Grinstein H., 1976. Solar heating by polyethylene mulching for the control of diseases caused by soilborne pathogens. Phytopathology, 66, 683-688.

Kimball B. A., 1973. Simulation of the energy balance of a greenhouse. Agric. Meteorol., 11, 243-260.

Kirkham D. \& Powers W. L., 1972. Advanced soil physics. Wiley, New York, 534 p.

Liakatas A., Clark J. A., Monteith J. L., 1986. Measurements of the heat balance under plastic mulches. Part $\mathbf{I}$. Radiation balance and soil heat flux. Agric. For. Meteorol., 36, 227-239.

Mac Adams W. H., 1954. Heat transmission. McGraw-Hill, New York, $431 \mathrm{p}$.

Mahrer Y., 1979. Prediction of soil temperatures of a soil mulched with transparent polyethylene. J. Appl. Meteorol., 18, 1263-1267.
Mahrer Y., 1980. A numerical model for calculating the soil temperature regime under transparent polyethylene mulches. Agric. Meteorol., 22, 227-234.

Mahrer Y. \& Katan J., 1981. Spatial soil temperature regime under transparent polyethylene mulch : numerical and experimental studies. Soil Sci., 131, 82-87.

Mahrer Y., Naot O., Rawitz E., Katan J., 1984. Temperature and moisture regimes in soils mulched with transparent polyethylene. Soil Sci. Soc. Am. J., 48, 362-367.

Nisen A., 1969. L'éclairement naturel des serres. J. Duculot, Gembloux, $198 \mathrm{p}$.

Sinoquet H., 1985. Evaluation des composantes du rayonnement solaire en Guadeloupe. C.R. Programme Régional « Maîtrise de l'Energie ", Note interne I.N.R.A. Guadeloupe, 25 p.

Valmorin M., 1986. Utilisation d'un micro Husky Hunter pour le transfert de données entre un Logger CR2I et un micro Apple II. Note interne I.N.R.A. Guadeloupe, $11 \mathrm{p}$.

De Vries D. A., 1963. Thermal properties of soils, p. 210-235. In : W. R. Van Wijk. Physics of plant environment. North-Holland Publishing Co., Amsterdam, $382 \mathrm{p}$. 